\title{
A survey of pain during rehabilitation after acute spinal cord injury
}

\author{
PW New, TC Lim, ST Hill and DJ Brown \\ Spinal Injuries Unit, The Austin and Repatriation Hospital, Heidelberg, Victoria, Australia 3084
}

There has been little research on pain in the acute phase of spinal cord injury (SCI) rehabilitation. This study surveyed the pain experience and management strategies in such patients.

The subjects consisted of inpatients who were undergoing rehabilitation following their acute injury, and were assessed regarding the presence and type of any pain upon admission to the rehabilitation ward, and reviewed weekly during their stay. They were reassessed on reporting any new pain. Pain intensity was recorded on a Visual Analogue Scale. The maximum intensity of pain during admission was compared to that at discharge. All interventions directed at pain management were documented. Patients were reviewed one year after discharge regarding current pain experience.

Almost all of the patients $(n=23 ; 96 \%)$ experienced pain at some stage during their inpatient rehabilitation. Overall pain intensity for those patients with pain during inpatient admission decreased by the time of discharge. At the one year review however, pain intensity tended towards that seen on admission. The reasons for pain tending to increase after discharge were not apparent.

Neuropathic and Myofascial Pain Syndrome (MPS) were the most common types of pain experienced. A combination of pharmacological, interventional, physical and psychological approaches were used in pain management. At one year review, neuropathic pain remained common while MPS and orthopaedic pain had decreased.

Pain is a common and significant problem for many SCI patients and is a challenge for the treating team to manage.

Keywords: spinal cord injury; neuropathic pain; myofascial pain syndrome; pain management; chronic pain

\section{Introduction}

Although pain is a recognised complication of spinal cord injury (SCI), the major focus of studies has been on either chronic pain ${ }^{1-4}$ or war related SCI that involved open spinal injuries. ${ }^{5-7}$ The incidence of chronic pain in these patients has been reported to be between $18 \%$ and $94 \%$. There has been little research on pain in the acute phase of acute SCI.

One study conducted spot surveys on three occasions, in two separate acute SCI units in Australia and USA. $^{8}$ The incidence of 'significant pain' was estimated from the frequency of pain complaints and medication use. No direct patient contact was involved. Considerable variability existed in the incidence of 'significant pain' (20\% and 59\%) and the interval after onset of SCI $(47 \%$ and $68 \%$ respectively were more than 3 months after SCI). Many of these patients had established chronic pain.

Another study evaluated pain in 40 SCI inpatients using a self-rating pain measurement technique and

Correspondence: Dr PW New: c/o Victorian Spinal Cord Service, Austin Campus, Austin and Repatriation Medical Centre, Victoria, Australia, 3184 activity checklist. ${ }^{9}$ The authors found that $60 \%$ reported 'significant pain' and decreased activity, and $25 \%$ used narcotic medications. In a comparison group of 24 outpatients, only $16.6 \%$ had 'significant pain' and decreased activity and only one patient required narcotics.

Most previous studies concerning the management of SCI pain have focused on one method or approach. The use of transcutaneous electrical nerve stimulation (TENS) for the treatment of musculoskeletal pain in the acute setting has been reported. ${ }^{10}$ Dysasthetic pain during acute SCI rehabilitation has been treated with a combination of amitriptyline and carbamazepine. ${ }^{11}$ Another study considered diagnostic categories of radicular, deafferentation and musculoskeletal pain. ${ }^{3}$ Various combinations of amitriptyline, clonazepam, nonsteroidal antiinflammatory drugs (NSAIDS), 5OH-tryptophane, TENS, spinal cord stimulation and continuous intrathecal morphine infusion were used in a step-wise progression and the outcomes reported.

Surgical management has also been described for the treatment of chronic pain when conservative methods are ineffective. ${ }^{12}$ The results however, are not conclusive and selection criteria are still being 
developed. ${ }^{13,14} \mathrm{~A}$ recent review has discussed medical, physical and surgical approaches to the management of chronic SCI pain. ${ }^{15}$

Psychological factors have been identified as being important in chronic SCI pain. ${ }^{16}$ It has been argued that these factors have been largely ignored by clinicians and researchers until recently. ${ }^{17}$

This study sought to define the type and severity of pain experienced by acute SCI inpatients during the period of active rehabilitation, and document the strategies used in the management of the pain conditions encountered. The study group was then surveyed a minimum of 12 months after discharge.

\section{Methods}

Patients sustaining an acute SCI were managed according to the Austin and Repatriation Hospital Spinal Injuries Unit Admissions Protocol. If required, they were admitted to the intensive care unit. An intravenous line was inserted for fluid, analgesics and medications. A suprapubic catheter and nasogastric tube were inserted, and calf stimulators applied. Patients sustaining cervical spine injuries were initially stabilised by head-tong traction before having definitive orthopaedic management. All patients were managed on ENGRIT electric turning beds (Engrit, Melbourne, Australia) and given pressure relief turns. As their medical condition stabilised patients were transferred to the acute ward, and subsequently to the rehabilitation unit to commence their formal rehabilitation program. The same medical staff were involved in the management of patients in both the acute medical and rehabilitation wards.

Pain management in the acute setting included intravenous morphine, sometimes using Patient Controlled Analgesia. Oral morphine was used when necessary. Psychological support was provided to assist patients with adjustment to their injury and in pain management. This support included the involvement of the liaison psychiatry registrar attached to the Unit.

For a 6 month period from 31 January 1994, all inpatients entering active rehabilitation after their acute injury were followed by the principal author. Patients were assessed regarding the presence and type of pain within $48 \mathrm{~h}$ of transfer to the rehabilitation ward. They were reviewed at least weekly during their stay until discharge. Patients were reassessed upon reporting of any new pain, or significant increase in existing pain. Patients were routinely reviewed by the Unit in an outpatient clinic 1, 3, 6 and 12 months after discharge.

A slide rule Visual Analogue Scale (VAS) was used by patients to indicate their perceived pain intensity. Some tetraplegic patients were unable to use adaptive devices to position the slide marker in the position that corresponded to their perceived pain intensity. For these patients, the marker was positioned at no pain and they verbally indicated how far it should be moved.
Pain intensity was recorded at the time of transfer or diagnosis of pain, when reassessed, and within $48 \mathrm{~h}$ prior to discharge. The maximum VAS during each admission (Admission MAX) was compared to that at discharge.

Neuropathic pain was diagnosed on the basis of the pain quality; burning, stabbing, pins and needles or numbness in nature; and located at or distal to the level of paralysis. ${ }^{18}$

When diagnosing MPS, a broader application of the usual diagnostic criteria ${ }^{19}$ was used. We included patients who failed to demonstrate the typical pattern of pain radiation associated with trigger point stimulation, but met all other major criteria.

Orthopaedic pain was either secondary to fractures sustained in the accident causing the SCI, or involved orthopaedic sources of pain that developed after the SCI.

All pain conditions detected in patients were recorded. Patients were investigated where appropriate to confirm the diagnosis or exclude other pathologies.

Pain management involved the patient and all members of the treating team in an interdisciplinary approach. This included medical, nursing, physiotherapy, occupational therapy, clinical psychology and psychiatric staff. More conservative treatment approaches were used first. A combination of pharmacological, physical, interventional and cognitivebehavioural psychological approaches ${ }^{20,21}$ was used to manage patients' pain. All pain management strategies used were noted. No formal measurement of strategy efficacy was undertaken. The diagnosis of pain category and management strategies used were supervised by the Unit consultants.

Oral analgesics were used in pain management. In patients with severe neuropathic pain not responding to antidepressants and anticonvulsants, trials of intravenous phentolamine and lignocaine were conducted. If patients responded to either of these infusions, a trial of oral Prazosin or Mexilitine was given. $^{22-24}$

Physiotherapy, NSAIDs and dry needling were used to treat MPS. Sympathetic ganglion nerve blocks were used to treat pain associated with Complex Regional Pain Syndrome Type II (CRPS II).

Patients were interviewed over the telephone between 13 and 16 months after discharge. The diagnostic category of any SCI related pain was determined. They were asked to score the maximum intensity of pain over the previous week between zero and 10 and they were asked about their current pain management strategies. See appendix.

Descriptive analysis was performed on the data.

\section{Results}

\section{Patient characteristics}

There were $22(92 \%)$ males and $2(8 \%)$ females followed from admission to discharge. Most patients 
$(n=22 ; 92 \%)$ were interviewed 1 year after discharge. The average age was 41.2 years (range 15 to 76 ). The average length of stay (LOS) on the acute ward before transferring to the rehabilitation ward was 31 days (range 12 to 76). The average LOS for inpatient rehabilitation was 117 days (range 35 to 261). Most injuries $(n=19 ; 79 \%)$ resulted from trauma. Most patients $(n=15 ; 63 \%)$ had an incomplete SCI (ASIA B, C or D). Tetraplegia was present in $54 \%$ of patients, and $34 \%$ had a conus or cauda equina lesion. Acute orthopaedic management involved open reduction and internal fixation, with or without bone grafting in 12 patients. Non-operative management using external stabilisation devices was used in seven patients.

\section{Pain experience}

Neuropathic pain was the most common pain category recorded during inpatient rehabilitation and its incidence had decreased by discharge. By 1 year review however, neuropathic pain had returned to the inpatient frequency (Figure 1). Two patients had complete resolution of neuropathic pain during their inpatient rehabilitation. At 1 year review there were another two patients whose neuropathic pain had fully resolved. Four patients however, who had no neuropathic pain during inpatient rehabilitation, had this diagnosis at review.

During inpatient rehabilitation one patient with neuropathic pain was found to have post-traumatic syringomyelia on Magnetic Resonance Imaging and subsequently had a shunt procedure performed. No other patients with post-traumatic syringomyelia had been detected by 1 year review. One patient developed CRPS II during inpatient rehabilitation that contributed to a frozen shoulder. One patient with severe neuropathic pain had his inpatient rehabilitation program interrupted for 35 days because he required transfer back to the acute ward for investigation and management of his neuropathic pain.

MPS declined during inpatient rehabilitation and this trend continued after discharge.

Orthopaedic pain was not common beyond early inpatient rehabilitation. Of the patients with orthopaedic pain, three were due to associated fractures incurred at the time of the SCI. At review, one patient reported persisting pain at the fracture site. This was not associated with neurological deterioration. During rehabilitation one paraplegic patient developed shoulder pain that was due to overuse. It was not associated with MPS and settled with conservative management. At 1 year review, a different patient had shoulder pain reportedly due to overuse. External stabilisation devices were associated with pain in three patients during inpatient rehabilitation. This pain was severe $(\mathrm{VAS}=8.0)$ in only one case. In all of these patients the pain was adequately managed by an adjustment of the device in question and primary analgesics. One patient developed painful heterotrophic ossification during inpatient rehabilitation, but this was not painful at review.

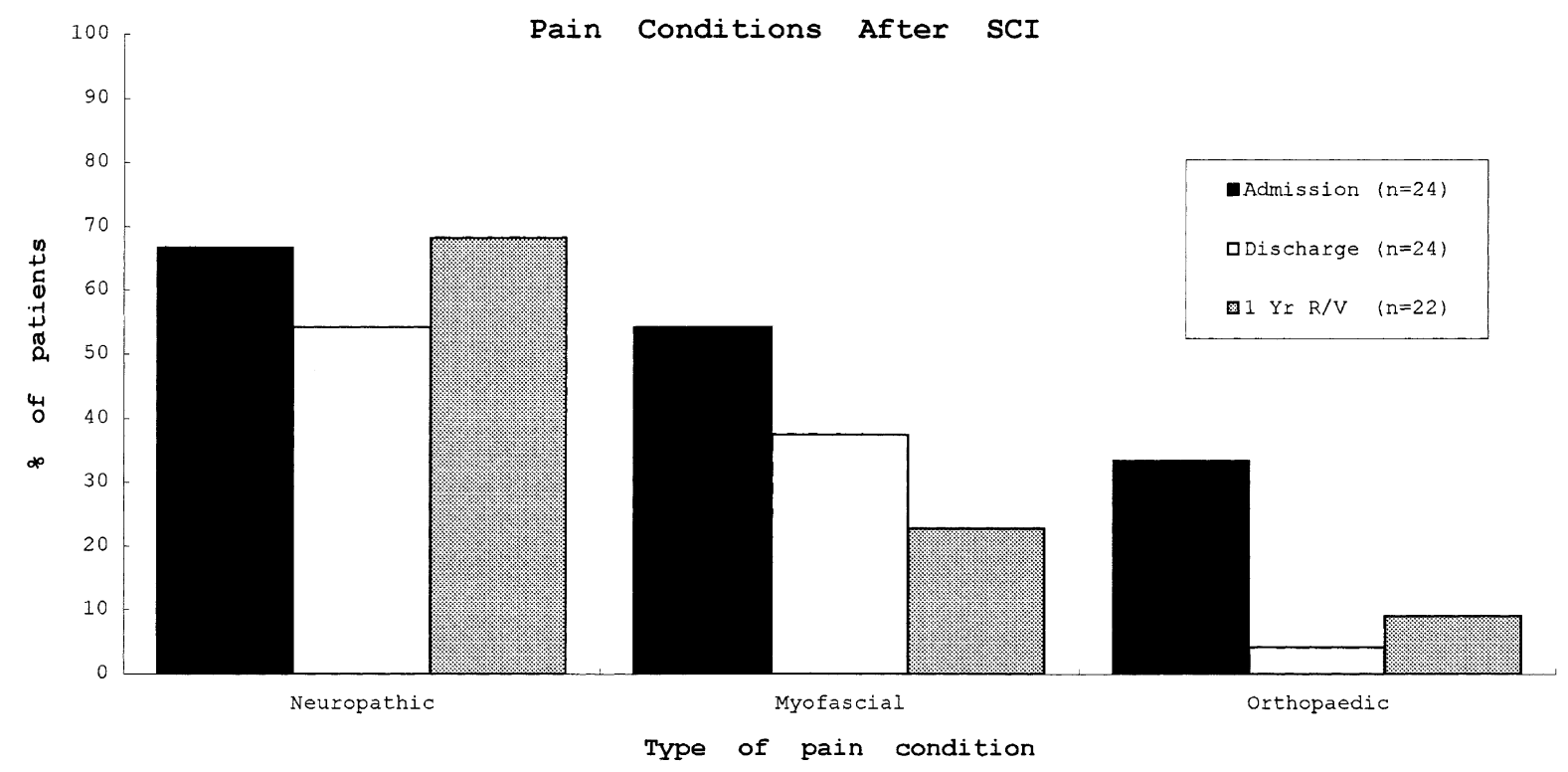

Figure 1 Pain conditions after SCI. 
No patient had pain that was due to radicular or mechanical sources.

Only one patient $(4 \%)$ reported no pain during inpatient rehabilitation. There were five patients $(21 \%)$ who reported being free of pain at discharge. At 1 year review there were two patients $(9 \%)$ who were pain free.

All patients with pain during their inpatient admission had a reduction in intensity by the time of discharge. In patients with neuropathic pain, 79\% reported greater than $50 \%$ reduction in pain intensity. In patients with MPS, $85 \%$ reported greater than $50 \%$ reduction in pain intensity. By the 1 year review however, pain intensity tended towards that seen during admission and $32 \%$ of patients reported pain of equal or greater intensity (Table 1). Although the overall intensity of pain at discharge was less than the maximum during admission, the intensity of pain was highly variable as indicated by the wide range and high standard deviation. This variability was also seen in the one year review results. The same pattern of change in pain intensity was seen with individual pain categories.

Of the patients reporting pain during inpatient rehabilitation, $48 \%(n=23)$ had multiple pain conditions. At 1 year review only $10 \%(n=20)$ of patients with pain reported multiple pain conditions.

\section{Pain management}

Patients received a combination of pharmacological, physical, interventional and psychological approaches for pain management. The frequency of different pain management strategies is shown in Table 2. Primary analgesics and co-analgesics were used in $91 \%$ of patients with pain during inpatient rehabilitation. The most common agents used were paracetamol and combination preparations with codeine $(63 \%)$, antidepressants $(41 \%)$, vitamin B and thiamine $(36 \%)$,

Table 1 Summary of changes in pain intensity

\begin{tabular}{|c|c|c|c|}
\hline & Admission & Discharge & 1 Year Review \\
\hline verage VAS & 7.32 & 2.74 & 5.90 \\
\hline Standard Deviation & 1.33 & 2.04 & 2.73 \\
\hline Range VAS & $4-9$ & $0-7$ & $0-9$ \\
\hline
\end{tabular}

prazosin $(32 \%)$ and anticonvulsants (32\%). No patient was receiving narcotic analgesics on admission to the rehabilitation ward. Only one patient $(5 \%)$ received narcotics during inpatient rehabilitation, but was not receiving them at discharge. TENS was used on eight patients $(36 \%)$ and ultrasound on seven patients $(32 \%)$. Dry needling was used in $59 \%$ of patients. Overall, $74 \%$ of inpatients had more than one pain management strategy used.

None of the patients surveyed at the 1 year review voluntarily reported themselves to be using psychological strategies to manage their pain. By further specific questioning the author determined that these strategies were being used by some patients. The exact frequency was not assessable because of the limitations of the telephone interview.

\section{Discussion}

The patient characteristics of this sample are typical of many other SCI rehabilitation centres. The sample size was small and this limited statistical analysis. For this reason no results were given for the changes in pain intensity for specific pain categories.

The measurement of pain in this study only considered the patients' perception of their pain intensity using the VAS. It is acknowledged that no measures of the pain quality or objective aspects of the pain experience were documented. The dynamic process of adjustment and psychological state would undoubtedly have influenced pain perception in the study group.

The VAS slide rule was used to measure pain in patients during admission, while at the 1 year review patients gave a verbal report of their perceived pain intensity. This may not be a valid method for comparing pain intensities. Patients were surveyed over the telephone because $46 \%$ lived more than 200 kilometres from the hospital and logistical factors would have made direct interview impractical. The 1 year review considered pain over the previous week. This period of time was used because the impact of pain on patients' would not be fully reflected by the reporting of pain occurring only at the time of questioning.

Neuropathic pain was the most common type of pain, and the most difficult to treat. There appeared to

Table 2 Pain management strategies

\begin{tabular}{|c|c|c|c|c|c|}
\hline & $\begin{array}{c}\text { Primary } \\
\text { analgesics }\end{array}$ & co-Analgesics & $\begin{array}{l}\text { Physical } \\
\text { therapies }\end{array}$ & $\begin{array}{l}\text { Interventional } \\
\text { therapies }\end{array}$ & $\begin{array}{c}\text { Psychological } \\
\text { strategies }\end{array}$ \\
\hline $\begin{array}{c}\text { Admission } \\
\quad(n=22)\end{array}$ & $68 \%$ & $59 \%$ & $36 \%$ & $64 \%$ & $59 \%$ \\
\hline $1 \begin{array}{l}\text { YR RV } \\
(n=20)\end{array}$ & $40 \%$ & $15 \%$ & $10 \%$ & $10 \%$ & not assessable \\
\hline
\end{tabular}

Primary analgesics $=$ paracetamol \pm codeine, NSAIDS, morphine. Co-analgesics $=$ antidepressants, anticonvulsants, vitamin $\mathrm{B}_{12}$ and thiamine, prazosin, mexilitine. Physical therapies $=$ TENS, Ultrasound. Interventional therapies = Dry needling, Sympathetic ganglion block 
be a continuation of neuropathic pain as a significant problem for most SCI patients beyond the acute phase. This is in contrast to the conclusion of other investigators. ${ }^{25}$ Further research is needed into the use of pharmacological agents, including intrathecal delivery, in the management of neuropathic pain.

MPS may present differently in SCI patients. Although the patients that we treated as MPS did not meet all diagnostic criteria, many seemed to respond to appropriate management strategies. Because of damaged neurological pathways in SCI patients the pain radiation typically described in this condition may not be seen. MPS was still a problem for some patients at 1 year review. Factors such as posture, immobility and overuse may contribute to this situation.

An eclectic approach is required to treat pain after SCI. ${ }^{15}$ Rehabilitation team members in SCI centres need to adopt the same approach to pain management as that used in chronic pain programs, even though these patients do not exhibit the full range of psychosocial symptoms and behavioural changes associated with chronic pain syndromes. There are significant problems encountered in analysing the utility of individual pain management techniques within the context of an interdisciplinary team approach utilising varying combinations of treatment strategies.

There may be a number of reasons for pain intensity increasing after discharge. The increase may not be real if the verbal report of pain intensity by patients is not valid. The interaction of the treating team with the patient may be a factor in patients' reporting a decrease in pain intensity during their inpatient admission. After discharge this interaction decreases significantly.

Psycho-social stresses are important factors in chronic pain. These can increase in the first year after SCI and may contribute to the patients' pain. These factors were not examined in this study. Patient use of medications decreased by the 1 year review. This may be a factor in the increase of pain. Alternatively, this may reflect a failure of medications to exert a therapeutic benefit. The reasons for patients failing to acknowledge their use of psychological strategies were not apparent. The use of these techniques in SCI outpatients needs further investigation.

Pain has an important impact on the quality of life for SCI patients. If the pain experience can be reduced, patient disability and quality of life can be positively influenced.

\section{Conclusion}

Pain is a common and significant problem for most acute SCI patients during their inpatient rehabilitation. This is a challenge for both the patient and treating team to manage. Utilising an interdisciplinary approach, and a variety of therapeutic interventions, it is possible to reduce the pain experience to an acceptable level for most of these patients by discharge.
At the 1 year review, pain intensity had significantly increased for most patients. The reasons for this increase were not examined in this study and require further investigation. Neuropathic pain is the most persistent and difficult type of pain to treat, and further research is needed to determine the most appropriate therapeutic interventions.

\section{Acknowledgements}

The authors would like to thank the staff and patients of the Austin and Repatriation Hospital Spinal Injuries Unit for their participation in this research.

\section{References}

1 Rose M, Robinson JE, Ells P, Cole JD. Pain following spinal cord injury: results from a postal survey. Pain 1988; 34: $101-102$.

2 Nepomuceno C et al. Pain in patients with spinal cord injury. Arch Phys Med Rehabil 1979; 60: 605-609.

3 Fenollosa $\mathrm{P}$ et al. Chronic pain in the spinal cord injured: statistical approach and pharmacological treatment. Paraplegia 1993; 31: $722-729$.

4 Woolsey RM. Chronic pain following spinal cord injury. $\mathrm{J} \mathrm{Am}$ Paraplegic Soc 1986; 9: $39-41$.

5 Davis L, Martin J. Studies upon spinal cord injuries: nature and treatment of pain. J. Neurosurg 1947; 4: 483-491.

6 Botterell EH, Callaghan JC, Jousse. Pain in paraplegia: clinical management and surgical treatment. Proc R Soc Med 1954; 47: $281-288$.

7 Bors E. Management of chronically ill patient. In: Whol MG (ed). Longterm Illness: management of the chronically ill patient. WB Saunders: Philadelphia 1959, pp 469-480.

8 Burke DC. Pain in paraplegia. Paraplegia 1973; 10: 297-313.

9 Lamid S, Chia JK, Kohli A, Ernesto C. Chronic pain in spinal cord injury: comparison between inpatients and outpatients. Arch Phys Med Rehabil 1985; 66: 777 - 778 .

10 Richardson RP, Meyer PM, Cerullo LJ. Transcutaneous electrical neurostimulation in musculoskeletal pain of acute spinal cord injuries. Spine 1980; 5: $42-45$.

11 Sandford P, Lindbom LB, Haddox JD. Amitriptyline and carbamazepine in the treatment of dysesthetic pain in spinal cord injury. Arch Phys Med Rehabil 1992; 73: 300-301.

12 Livshits AV. The algesic syndrome in spinal cord trauma. Paraplegia 1992; 30: $497-501$.

13 Sampson JH, Cashmore RE, Nashold BS Jr, Friedman AH Dorsal root entry zone lesions for intractable pain after trauma to the conus medullaris and cauda equina. Journal of Neurosurg 1995; 82: $28-34$.

14 Cioni I, Meglio M, Pentimilli L, Visocchi M. Spinal cord stimulation in the treatment of paraplegic pain. Journal of Neurosurg 1995; 82: 35-39.

15 Balazy TE. Clinical management of chronic pain in spinal cord injury. The Clinical Journal of Pain 1992; 8: 102-110.

16 Summers JD et al. Psychosocial factors in chronic spinal cord injury pain. Pain 1991; 47: $183-189$.

17 Mariano AJ. Chronic pain and spinal cord injury. The Clinical Journal of Pain 1992; 8: 87-92.

18 Frisbie JH, Aguilera EJ. Chronic pain after spinal cord injury: an expedient diagnostic approach. Paraplegia 1990; 28: 460-465.

19 Travell JG, Simons DG. Myofascial pain and dysfunction: the trigger point manual. WB Saunders Williams \& Wilkins: Baltimore, 1983.

20 Turk DC, Meichenbaum D, Genest M. Pain and behavioural medicine: a cognitive behavioural perspective. Guildford: New York, 1983.

21 Turner JA, Chapman CR. Psychological interventions for chronic pain: a critical review. Pain 1983; 13: $23-46$. 
22 Edmondson EA, Simpson KR Jr, Stubler DK, Beric A. Systemic lidocaine therapy for poststroke pain. Southern Medical Journal 1993; 86: $1093-1096$.

23 Tanelian DL, Brose WG. Neuropathic pain can be relieved by drugs that are use-dependent sodium channel blockers: lidocaine, carbamazepine and mexilitine. Anasthesiology 1991; 74: 949 951.

24 Raja SN, Treede RD, Davis KD. Systemic alpha-adrenergic blockade with phentolamine: a diagnostic test for sympathetically maintained pain. Anasthesiology 1991; 74: 691-698.

25 Cairns DM, Adkins RH, Scott MD. Pain and depression in acute traumatic spinal cord injury: Origins of chronic problematic pain? Arch Phys Med Rehabil 1996; 7: 329-335.

\section{Appendix}

Telephone Interview

1 Have you had any pain in the past week caused by your spinal cord injury?

2 At your last spinal outpatients review, to what were you told this pain was due?
A brief description was obtained to confirm the category according to the classification below. This classification was also used if the pain onset was subsequent to the last review:

Neuropathic pain: Burning, stabbing, pins and needles or numbness in nature and located at or distal to the level of paralysis.

Myofascial pain: Aching pain in muscles exacerbated by firm pressure.

Orthopaedic pain: Pain in joint(s) or at previous fracture sites.

3 On a scale of zero to ten, where zero is no pain at all and ten the worst pain you have ever experienced, what number would you put on the maximum intensity of pain that you have experienced in the past week?

4 What are you currently doing to manage your spinal cord injury related pain? 